\title{
Simultaneously Reducing Cost and Improving Performance of NVM-based Block Devices via Transparent Data Compression
}

\author{
Xubin Chen, Yin Li \\ Jingpeng Hao \\ Renssealer Polytechnic Insitute \\ chenx22@rpi.edu
}

\author{
Hyunsuk Shin, Michael Suh \\ Qualcomm \\ \{hyunsuks,jsuh\}@qti.qualcomm.com
}

\author{
Tong Zhang \\ Renssealer Polytechnic Insitute \\ tzhang@ecse.rpi.edu
}

\begin{abstract}
This paper studies the integration of data compression into block devices built upon non-volatile memory (NVM). Although byteaddressable NVM-based main memory systems received most attention from the research community, industry chose to first commercialize NVM-based block devices, at least partly because block devices can much better mitigate non-ideal NVM characteristics (e.g., random bit errors, wear-out, and defects). It is very likely that NVMbased memory and block devices will co-exist in future computing systems, complementing to DRAM and flash memory. Compared with their flash-based counterparts, NVM-based block devices have shorter read latency but suffer from higher cost. Although data compression can reduce storage cost, conventional wisdom suggests that data compression inevitably degrades the access latency. Hence one may intuitively conclude that NVM-based block devices with built-in data compression must trade latency for bit cost. This paper shows that such an intuitive conclusion is not necessarily true. In particular, this paper presents a set of architectural design techniques that can reduce the read latency of NVM-based block devices with built-in data compression. Simulation results show that, compared with NVM-based block devices without built-in data compression, we can significantly reduce the read latency (e.g., by even more than $90 \%$ ) without compromising the capability of tolerating non-ideal NVM characteristics.
\end{abstract}

\section{CCS CONCEPTS}

- Hardware $\rightarrow$ Non-volatile memory; Memory and dense storage; • Information systems $\rightarrow$ Storage class memory; Data compression; - Computer systems organization $\rightarrow$ Reliability.

\section{KEYWORDS}

Non-Volatile Memory (NVM), Block Devices, Data Compression, Error Correction Code (ECC), Cost, Performance, Latency

\section{ACM Reference Format:}

Xubin Chen, Yin Li, Jingpeng Hao, Hyunsuk Shin, Michael Suh, and Tong Zhang. 2019. Simultaneously Reducing Cost and Improving Performance of NVM-based Block Devices via Transparent Data Compression. In Proceedings of the International Symposium on Memory Systems (MEMSYS '19),

Permission to make digital or hard copies of all or part of this work for personal or classroom use is granted without fee provided that copies are not made or distributed for profit or commercial advantage and that copies bear this notice and the full citation on the first page. Copyrights for components of this work owned by others than ACM must be honored. Abstracting with credit is permitted. To copy otherwise, or republish, to post on servers or to redistribute to lists, requires prior specific permission and/or a fee. Request permissions from permissions@acm.org.

MEMSYS '19, September 30-October 3, 2019, Washington, DC, USA

(c) 2019 Association for Computing Machinery.

ACM ISBN 978-1-4503-7206-0/19/09 . \$ \$15.00

https://doi.org/10.1145/3357526.3357530
September 30-October 3, 2019, Washington, DC, USA. ACM, New York, NY, USA, 11 pages. https://doi.org/10.1145/3357526.3357530

\section{INTRODUCTION}

This paper studies the architectural design of block devices built upon highly scalable non-volatile memory (NVM) technologies (e.g., 3DXP and ReRAM). Motivated by the fine-grained accessibility (e.g., 32B or 64B per access) of NVM chips, prior research focused on NVM-based main memory systems (e.g., see [16, 28, 32, 34, 38]). In comparison, NVM-based block devices received little attention. Nevertheless, it is evident that block devices can much more easily accommodate the non-ideal NVM characteristics (e.g., random bit errors, defects, limited cycling endurance, and process variations). Therefore, not surprisingly the industry first commercialized NVMbased products in the form of block devices (i.e., Intel Optane NVMe SSD). It is plausible that future NVM-based products will exist in the form of both byte-addressable devices and block devices, representing different cost vs. performance trade-offs and residing at different layers along the memory/storage hierarchy.

Compared with their flash-based counterparts, NVM-based block devices have much shorter read latency (e.g., $\sim 10 \mu$ s of Optane SSD vs. $>50 \mu$ s of MLC/TLC SSDs). With internal SRAM/DRAM write buffer with power loss protection, block devices (either NVM-based or flash-based) always exhibit similar write latency (e.g., $\sim 10 \mu \mathrm{s})$. Nevertheless, NVM suffers from much higher bit cost than NAND flash memory, at least in the foreseeable future. Intuitively, in addition to technology scaling, data compression may contribute to lowering the cost barrier and hence expanding the addressable market of NVM-based block devices. However, conventional wisdom suggests that the use of compression always accompany with extra data read latency. Since the main advantage of NVM-based block devices is short read latency, one may be justifiably skeptical about the practical feasibility of applying data compression to reduce the bit cost of NVM-based block devices.

This paper shows that, for NVM-based block devices, compression does not necessarily incur latency penalty. This paper presents techniques that can even noticeably reduce data read latency, compared with not using compression. This work focuses on built-in transparent data compression, i.e., block devices internally handle data compression with complete transparency to host. Hence, block devices can maintain the benefits of data compression no matter whether they are used as I/O devices (e.g., NVMe SSD) or block devices inside hybrid memory modules (e.g., NVDIMM-P).

In order to best embrace the non-ideal NVM characteristics, block devices must employ strong fault tolerance. Each NVM physical page (e.g., $512 \mathrm{~B}$ or $4 \mathrm{kB}$ ) must be protected by a strong error correction code (ECC) with a long codeword length. As shown 
later in Section 2.1, a long ECC codeword length (e.g., 4kB) can enable orders-of-magnitudes stronger error correction than a short codeword length (e.g., 64B). To limit the impact of compression on read latency, we focus on constrained data compression with the following specific constraints: (1) each data block is compressed individually, and (2) each compressed block entirely resides in one physical page (and hence in one ECC codeword). Compared with options that can achieve better compression efficiency (e.g., apply compression across multiple data blocks, or allow one compressed block reside across two physical pages), the constrained data compression essentially trades bit cost reduction efficiency for lower data read latency. For our interested NVM-based block devices, it is very important to minimize read latency. Meanwhile, such constrained data compression can largely simplify the data management, which is highly desirable because of the very limited memory and processing resources inside block devices.

Under the framework of transparent constrained data compression, we developed four architectural design techniques to minimize the block device read latency: (1) Optimistic data decompression: It can hide the latency of data decompression on the data read path. The key is to optimistically integrate the ECC decoding and data decompression operations together. (2) Zero-padding with punctured ECC coding: It can reduce the physical page read latency by cohesively leveraging the fine-grained NVM read/write accessibility and unique properties of linear block ECC. (3) Data access locality aware compaction: Block devices with built-in transparent data compression must carry out background compaction operations to reclaim stale storage space, which may cause read latency overhead. Leveraging the data access locality in most real-world workloads, we present a compaction scheduling scheme to reduce its impact on read latency. (4) Intra-page delta encoding: Block devices without built-in data compression can leverage the fine-grained NVM data write accessibility to effectively handle data block overwrite with strong content locality (i.e., blocks with similar content are consecutively written to the same address). However, data compression makes it difficult to exploit the content similarity of data block overwrite. We propose an intra-page delta encoding scheme that can exploit the content similarity in the presence of transparent data compression.

To facilitate evaluation, we implemented an NVM-based block device simulator based upon DiskSim [3] with the SSD module [1], which has a multi-channel architecture in order to improve the internal operational parallelism. Moreover, it stripes each $4 \mathrm{kB}$ data block across two channels to further reduce the read latency. This work sets the total NVM sensing latency of each $4 \mathrm{kB}$ block as only $2 \mu \mathrm{s}$. For an 8 -channel NVM block device, this corresponds to $8 \mathrm{~GB} / \mathrm{s}$ internal NVM read throughput, which can saturate the PCIe Gen3x8 interface. This study assumes that $4 \mathrm{kB}$ data block NVM write latency can be as low as $4 \mu \mathrm{s} \sim 20 \mu$ s. Under a wide range of data reduction ratios and data access characteristics, we carried out simulations to estimate the data read latency. The results show that, compared with block devices without built-in data compression, the developed design techniques can achieve significant read latency reduction (e.g., by even more than $90 \%$ ) without degrading write latency. The results also reveal a large bit cost vs. read latency trade-off space enabled by the developed design techniques.

\section{MOTIVATION AND BACKGROUND}

\subsection{Why NVM-based Block Devices?}

After almost twenty years of intensive research on various NVM technologies, there is a growing consensus that, compared with DRAM, NVM technologies with good scalability (e.g., 3DXP and ReRAM) tend to have much longer access latency (i.e., at least $2 \sim 5 \times$ ) and significantly lower cycling endurance (i.e., by at least $3 \sim 5$ orders of magnitudes). Therefore, instead of replacing DRAM, NVM will reside between DRAM and flash memory, and may exist in two different forms: (1) NVM-based low-cost byte-addressable memory devices that complement with DRAM, and (2) NVM-based shortlatency block devices that complement with flash. NVM-based block devices could be used as standard I/O devices (e.g., NVMe SSDs) or reside inside hybrid memory module (e.g., NVDIMM-P).

Compared with NVM-based main memory systems, NVM-based block devices have received little attention from research community. Nevertheless, the industry chose to first commercialize NVMbased block devices (i.e., 3DXP-based Optane SSD). This indeed is not a surprise since block devices can much more conveniently employ powerful fault tolerance and sophisticated management to mitigate the non-ideal NVM characteristics (e.g., random bit errors, defects, limited cycling endurance, and process variations). For example, suppose we use $\mathrm{BCH}$ code as ECC, and fix the user data vs. ECC redundancy ratio as 16:1. Fig. 1 shows the $\mathrm{BCH}$ code decoding failure rate vs. raw bit error rate under different codeword length. In order to achieve the decoding failure of $\sim 10^{-20}$, the $\mathrm{BCH}$ code with $64 \mathrm{~B}$ user data per codeword can only tolerate the bit error rate of $4 \times 10^{-8}$. By increasing the amount of user data per codeword to $4 \mathrm{kB}$, we could tolerate the bit error rate of $1.8 \times 10^{-3}$, representing almost five orders of magnitudes of improvement on the error tolerance. Moreover, block devices can employ sophisticated management algorithms to much better embrace the limited cycling endurance and defects. These factors make NVM-based block devices have (much) lower bit cost than NVM-based byteaddressable devices. Meanwhile, compared with flash-based block devices, NVM-based block devices have much lower read latency. This can translate into significant performance benefits for many real-life systems.

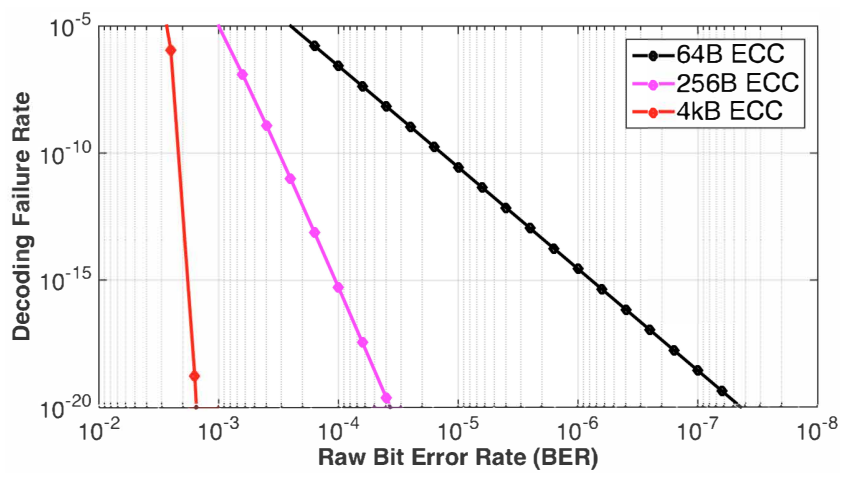

Figure 1: The ECC decoding failure rate vs. raw bit error rate under different codeword length when using $\mathrm{BCH}$ codes as ECC with the user data vs. coding redundancy ratio of 16:1. 


\subsection{ECC inside NVM-based Block Devices}

As one widely used ECC, $\mathrm{BCH}$ code can achieve excellent error correction strength with low encoding/decoding complexity. Hence, this work considers BCH-based ECC inside NVM-based block devices. Given an $(n, k, t) \mathrm{BCH}$ code, which protects $k$-bit user data using $(n-k)$-bit redundancy and can correct up to $t$ random errors, its decoding consists of the following four tasks [2] (as illustrated in Fig. 2):

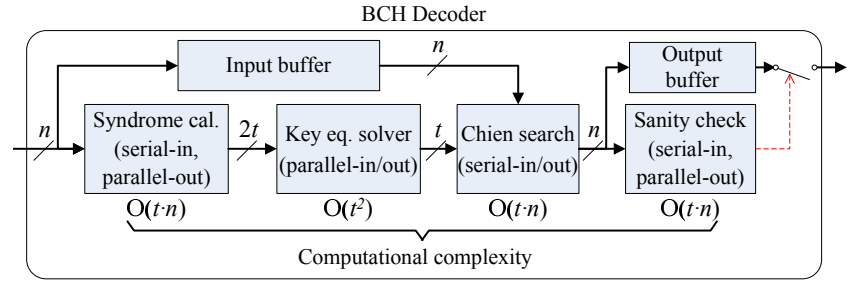

Figure 2: Four major computational components of $\mathrm{BCH}$ code decoding.

(1) Syndrome calculation: $\mathrm{BCH}$ decoder first calculates $2 t$ syndromes by sequentially processing the $n$-bit raw data fetched from memory chips. Its computational complexity is proportional to $t \cdot n$.

(2) Key equation solver: Upon the $2 t$ syndromes, the $\mathrm{BCH}$ decoder formulates and solves a so-called key equation. With the computational complexity proportional to $t^{2}$, this step is implemented in a partially parallel architecture that can finish the computation in $t$ clock cycles.

(3) Chien search: This step evaluates the key equation over all the bit positions to find the errors. Whenever one bit position renders a zero for the key equation evaluation, this bit position is marked as an error and corrected by a bit flipping.

(4) Decoding sanity check: Before it sends out the decoding output, the decoder must verify the integrity of the results. This is because Chien search may produce an invalid $\mathrm{BCH}$ codeword if more than $t$ errors occur. Let $(\hat{\mathbf{U}}, \hat{\mathbf{R}})$ represent the user data and redundancy produced by Chien search. We re-encode $\hat{\mathbf{U}}$ to generate its coding redundancy, denoted as $\tilde{\mathbf{R}}$. BCH decoding sanity check fails if $\tilde{\mathbf{R}}$ is not equal to $\hat{\mathbf{R}}$.

If the sanity check fails, block device controller re-issues the NVM read with adjusted memory sensing configurations, and repeat the ECC decoding. If the failure persists, the block device reports a read failure to the host.

\subsection{Read Latency Estimation}

When block devices serve a read request, besides the queuing latency, the physical read latency mainly includes the latency of storage media sensing, data transfer, and ECC decoding. For flashbased block devices, flash memory sensing latency is significantly longer than the latency of data transfer and decoding (e.g., $>50 \mu \mathrm{s}$ vs. a few $\mu$ s). In comparison, for NVM-based block devices, memory sensing latency becomes comparable to the latency of data transfer and decoding. Hence, we must take into account all the three major operations to accurately estimate the physical read latency.

As pointed out above in Section 2.2, the first step of $\mathrm{BCH}$ decoding (i.e., syndrome calculation) sequentially consumes the input bits.
It can overlap with the sequential byte-by-byte NVM-to-controller data transfer, and hence does not add extra latency on the read path. The last two steps (i.e., Chien search and sanity check) can operate concurrently, because of their serial processing nature. Since NVM on-chip memory sensing is carried out on a batch of memory cells (e.g., 128 or 256) at a time, it can overlap with the NVM-to-controller data transfer. We can exploit the inter-operation concurrency to minimize the physical read latency, where Fig. 3 illustrates the corresponding time-domain allocation of different operations. In addition, as illustrated in Fig. 3, except the key equation solver whose latency is proportional to the error correction strength $t$, the latency of all the other operations is proportional to the codeword length $n$.

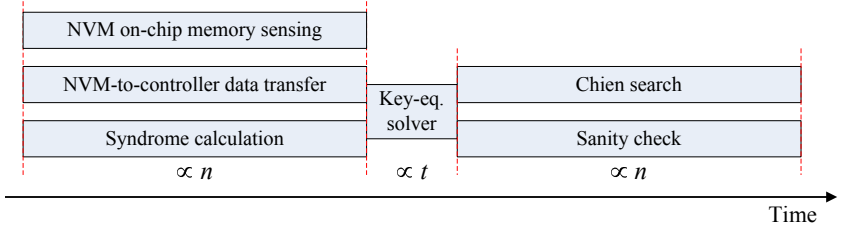

Figure 3: Illustration of the time-domain duration of different operations when reading one data block inside NVMbased block devices.

Let us further consider the following example. Assume the total memory sensing latency is only $2 \mu$ s per $4 \mathrm{kB}$ block, which is matched with $2 \mathrm{~GB} / \mathrm{s}$ NVM-to-controller data transfer bandwidth. Assume the use of a length- $4 \mathrm{kB} \mathrm{BCH}$ code with the code rate of $16 / 17$ (i.e., the user data vs. coding redundancy ratio is $16: 1$ ), its error correction strength $t$ is 128 . Assume the $\mathrm{BCH}$ decoder can operate at a clock frequency of $1 \mathrm{GHz}$. The key equation solver takes $t$ (i.e., 128) clock cycles, leading to $128 \mathrm{~ns}$ latency. Assume the other tasks process 16 input bits per clock cycle, corresponding to $2 \mu$ s latency of each task. Therefore, as illustrated in Fig. 3, we can estimate that the total physical read latency is about $4 \mu \mathrm{s}$, in which $2 \mu$ s is due to memory sensing and data transfer, and the other $2 \mu$ s is due to $\mathrm{BCH}$ decoding.

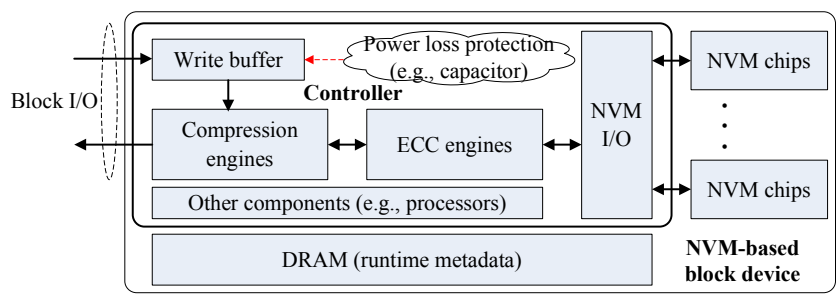

Figure 4: Architecture of NVM-based block devices with built-in compression.

\section{PROPOSED DESIGN SOLUTIONS}

Fig. 4 shows the architecture of NVM-based block devices with built-in compression. The controller integrates enough number of compression and ECC engines that can saturate the controllerNVM data transfer bandwidth. One or multiple processor cores 
inside the controller are responsible for all the firmware functions (e.g., address translation, defects management, and wear-leveling). The DRAM module stores runtime metadata (e.g., address mapping table).

Inside block devices, each physical page is protected by a $\mathrm{BCH}$ code and assigned with a unique physical block address (PBA). To limit the impact on read latency, compression is done with two constraints: (i) each data block is compressed individually, and (ii) each compressed block entirely resides in one physical page. To materialize the cost reduction, block devices expose a thin-provisioned logical block address (LBA) space, and each physical page stores one or multiple compressed blocks. Within one physical page, each compressed block has its own header that contains metadata including the LBA, time-stamp, and the length of the compressed block. The header serves for the following purposes: (1) The LBA and time-stamp information are used for crash recovery in case of mapping table corruption. (2) The LBA-PBA mapping table no longer needs to record the location of each compressed block inside the physical page, leading to a smaller mapping table. (3) It can help to skip the decompression of unwanted blocks, leading to energy saving. Under this architecture, the remainder of this section presents four design techniques that can reduce the data read latency for NVM-based block devices with built-in compression.

\subsection{Optimistic Data Decompression}

At the first glance, one may expect that data decompression inevitably increases the data read latency. If we follow the conventional practice of keeping the design of $\mathrm{BCH}$ decoder and data decompression engine completely separate, as illustrated in Fig. 5(a), data decompression indeed introduces read latency penalty, which could noticeably degrade the NVM-based block device read latency.

To obviate the decompression-induced read latency penalty, we propose to optimistically carry out decompression in parallel with the decoding sanity check, instead of waiting until the decoding sanity check finishes. This leads to the fused $\mathrm{BCH}$ decoding and decompression, as illustrated in Fig. 5(b). This design strategy can be justified by the following two factors: (1) Low failure probability of sanity check: In practice, the probability of sanity check failure should be very low (e.g., well below $1 \%$ even at the end of endurance lifetime of NVM). Hence, it is reasonable to be optimistic about the sanity check and hence move the decompression forward. (2) Serial-in serial-out data decompression: Data decompression consists of entropy code decoding and LZ reconstruction, both of which have a serial-in serial-out data processing flow. Therefore, data decompression can naturally operate in parallel with the decoding sanity check.

Once we implement the data decompression engine with the same data processing parallelism (e.g., processing 16 input bits per clock cycle) as the decoding sanity check, data decompression tends to finish at the same time as the sanity check. As a result, by fusing $\mathrm{BCH}$ decoding and data decompression, the proposed optimistic design strategy can eliminate the decompression-induced data read latency penalty. Moreover, as illustrated in Fig. 5(b), this design strategy enables the merge of the $\mathrm{BCH}$ decoder output buffer and block device output buffer, leading to a lower silicon implementation cost.
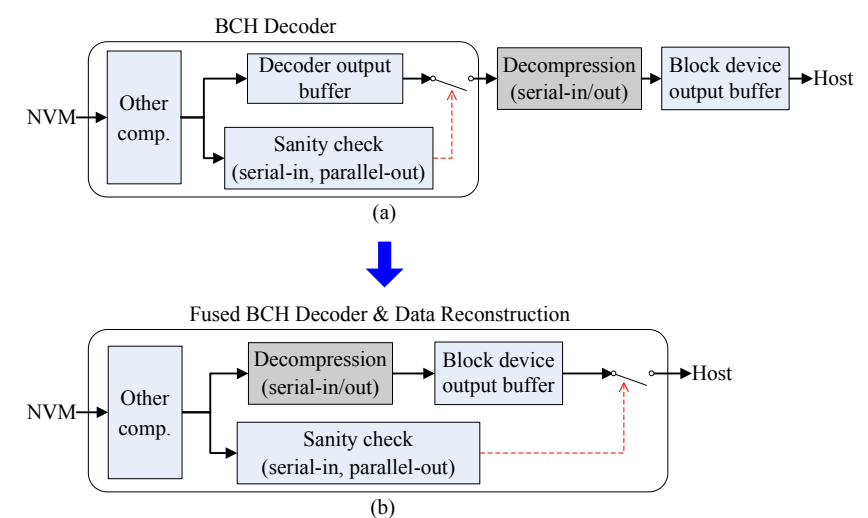

Figure 5: Architecture of (a) separate $\mathrm{BCH}$ decoder and data decompression as in conventional design practice, and (b) proposed optimistic data decompression by fusing $\mathrm{BCH}$ decoder and data decompression together.

\subsection{Zero-padding with Punctured ECC}

Because NVM chips support fine-grained data accessibility, the total sensing (or programming) latency is proportional to the amount of data being read (or written). Intuitively, one may expect that data compression should natively reduce read latency, not only because of less data to be read, but also because of less data to be written and hence less write-to-read interference. However, since each physical page is protected by a single long ECC codeword, accessing any data demands the decoding/encoding of the entire codeword. As a result, one may conclude that the use of strong ECC makes it impossible to take advantage of the fine-grained data accessibility of NVM to reduce read latency.

This work shows that, even in the presence of strong ECC, we can still leverage the fine-grained data accessibility of NVM to reduce the data access latency. The key is to utilize a simple concept called punctured ECC coding in the coding theory: When using an $(n, k, t)$ code to protect $\left(k-l_{u}\right)$-bit user data (where $\left.l_{u}>0\right)$, we can puncture the code to an $\left(n-l_{u}, k-l_{u}, t\right)$ code that still maintains the same $t$-error-correction strength. Logically, we may consider it as padding the original $\left(k-l_{u}\right)$-bit user data with $l_{u}$-bit zeros, and then apply the $(n, k, t)$ code. During the encoding and decoding, the codec can simply ignore (i.e., puncture) the $l_{u}$-bit all-zero segment. Once the block device controller keeps the record of $l_{u}$ for each physical page, it only fetches the $\left(k-l_{u}\right)$-bit real data and $(n-k)$-bit coding redundancy for punctured decoding, leading to a shorter read latency.

To implement this method, the storage device controller must know the value of $l_{u}$ for each physical page. During normal operation, we can keep all the $l_{u}$ 's in the DRAM inside block devices for fast access. Meanwhile, all the $l_{u}$ 's must be persistent through power failure and system crash. To persist $l_{u}$ 's, one simple solution is to directly write all-zeros to the unused space within each physical page, as illustrated in Fig. 6(a). This is called physical zeropadding. During the crash recovery, we can scan all the physical pages by reading/decoding each entire codeword and then parsing the headers of the compressed blocks to reconstruct the values of 
$l_{u}$ 's. However, the extra write activities of the physical zero-padding reduce NVM lifetime and cause more write-to-read interference.

\begin{tabular}{|c|c|c|}
\hline$\overbrace{\text { Compressed sector(s) }}^{4 \mathrm{kB}-l_{u}}$ & All-zero & $\begin{array}{c}\text { ECC } \\
\text { redundancy }\end{array}$ \\
\hline
\end{tabular}

$16 \mathrm{~B} \mathrm{BCH}$

\begin{tabular}{|c|c|c|}
\hline$l_{u}$ & Compressed sector(s) & $111010 \begin{array}{l}\mathrm{ECC} \\
\text { redundancy }\end{array}$ \\
\hline
\end{tabular}

Figure 6: Illustration of the two options for persisting $l_{u}$ 's: (a) intra-page physical zero-padding, and (b) intra-page virtual zero-padding.

To reduce the overhead, we propose a virtual zero-padding scheme. As illustrated in Fig. 6(b), the concept is simple: For each physical page, we attach a very small $\mathrm{BCH}$ codeword that only contains and protects the value of $l_{u}$. When writing a punctured $\mathrm{BCH}$ codeword to a physical page, we keep the unused storage space intact (i.e., without writing anything to the unused storage space), and meanwhile update the value of $l_{u}$ in the attached small $\mathrm{BCH}$ codeword. Since $l_{u}$ can be represented by less than 16 bits, a length-16B BCH codeword (i.e., with data vs. redundancy ratio of $1: 8)$ can easily guarantee the storage reliability even if the raw bit error rate is over $2 \%$. During normal operation, the storage device controller still keeps all the $l_{u}$ 's in the DRAM for fast access.

\subsection{Data Access Locality Aware Compaction}

Block devices with built-in transparent compression must carry out background compaction for two main purposes: (1) Data packing: pack multiple compressed blocks into one physical page to materialize the cost reduction, and (2) Garbage collection (GC): reclaim the storage space occupied by stale data blocks within physical pages. As we more aggressively carry out data packing, it may degrade the read latency from two aspects: (i) When using the zero-padding with punctured ECC, the more compressed blocks reside in one physical page, the longer latency it will take to read this physical page. (ii) Heavily packed physical pages are more subject to holes left by stale data, which leads to higher GC overhead. These two factors essentially lead to an inherent cost reduction vs. read latency trade-off.

This subsection presents a design strategy to achieve more desirable cost reduction vs. read latency trade-offs. The key is to exploit the data access locality in most real-world workloads. The basic concept is simple: If we more heavily pack relatively cold data together and meanwhile leave hot data in less packed physical pages, we could maintain short read latency without noticeably sacrificing cost reduction. In this context, we need to address two issues: (1) how to identify the hot data blocks, and (2) how to utilize the data identification results to enhance the background compaction process. Since prior work (e.g., see [6, 12, 17, 25]) has well studied the first issue, we only focus on the second issue.

As pointed out earlier, block devices always buffer incoming data first and then migrate them (after compression) to NVM chips in background. During buffer-to-NVM data migration, instead of packing multiple compressed blocks into one physical page, we propose to keep each compressed block in a separate physical page and postpone data packing to later compaction operations. This decision can be justified by the following two factors: (i) Most data classification methods rely on the data access history, and may not well handle freshly written data. We propose to optimistically treat all the freshly written data as hot data, and keep each one in an individual physical page. (ii) In many workloads, data overwrite occur over a relatively short period. If we immediately pack multiple compressed blocks into one physical page during buffer-to-NVM migration, subsequent data overwrite may cause holes in physical pages, leading to higher GC overhead. Moreover, as discussed later in Section 3.4, we can use intra-page delta encoding to reduce the NVM write activities for data overwrite with content similarity. Postponing data packing for freshly written data helps to increase the effectiveness of intra-page delta encoding.

During the runtime, the block device controller does not invoke the compaction process unless the number of empty physical pages falls below a certain threshold. Once being invoked, the compaction process cohesively performs data packing and garbage collection with the following two steps:

(1) Page classification: Let $\mathcal{G}$ denote the PBA set in which each physical page contains at least one stale compressed block. Within each physical page, compressed blocks are placed consecutively. We partition the set $\mathcal{G}$ into two subsets $\mathcal{G}_{h}$ and $\mathcal{G}_{t}$. The subset $\mathcal{G}_{h}$ contains all the physical pages that contain at least one hole (i.e., a stale compressed block resides in between two valid compressed blocks). The physical pages in the subset $\mathcal{G}_{t}$ do not contain any holes (i.e., the stale compressed blocks locate at the tail). Meanwhile, let $\mathcal{P}$ denote the PBA set in which each physical page only contains one compressed data block. By querying the data access intensity, we partition the set $\mathcal{P}$ into two subsets $\mathcal{P}_{h}$ and $\mathcal{P}_{c}$, where $\mathcal{P}_{h}$ contains all the hot (either write-hot or read-hot) data blocks, and $\mathcal{P}_{c}$ contains the rest.

(2) Selective data merging: After the page classification step, we keep the physical pages in the subset $\mathcal{P}_{h}$ (i.e., physical pages holding hot data blocks) intact, and pack the compressed data blocks being stored in physical pages in the subsets $\mathcal{G}_{h}$, $\mathcal{G}_{t}$, and $\mathcal{P}_{c}$. Since the physical pages in $\mathcal{G}_{h}$ contain holes, we first move all the compressed data blocks from physical pages in $\mathcal{G}_{h}$ into physical pages in the other two subsets, and subsequently mark all the physical pages $\mathcal{G}_{h}$ as empty pages. Then we further merge the data among all the physical pages in the subsets $\mathcal{G}_{t}$ and $\mathcal{P}_{c}$, which will make some physical pages more heavily packed and meanwhile produce more empty physical pages.

Finally, because the data access intensity may vary over the time, it is possible that a compressed block, which was classified as cold data and packed together with other blocks, becomes hot later on. To embrace the time-variant data access intensity, the storage device periodically scans all the packed physical pages that contain more than one compressed blocks. For each packed physical page that contains at least one hot compressed block, we move each hot compressed block to one empty physical page. As a result, we leave holes in those packed pages, and rely on the subsequent compaction process (as described above) to recycle the storage space occupied by holes. 


\subsection{Intra-page Delta Encoding}

In the case of data block overwrite with strong content similarity, intuitively we can leverage the fine-grained NVM write accessibility to reduce the NVM write activities [7, 35]. Meanwhile, prior work well demonstrated the existence of data overwrite with noticeable content similarity [4]. However, once data blocks are compressed, we cannot directly exploit the content similarity in data block overwrite. To address this issue, we propose an intra-page delta encoding technique: When serving a data block overwrite, in addition to the per-block data compression, we also evaluate the option of encoding the difference between the new and old content (i.e., delta encoding). To limit the impact on read latency, each data block and all its associated deltas must entirely reside in the same physical page. Moreover, to realize delta encoding, we must first fetch the old content from NVM, leading to data read overhead. Therefore, we should first carry out the compression, and bypass the delta encoding if the size of the compressed block is already very small.

The fine-grained NVM write accessibility enables flexible implementation of intra-page delta encoding, as illustrated by the following example. Suppose the host writes to the same LBA twice with the content $d_{1}$ and $d_{2}$. As shown in Fig. 7, $d_{1}$ is compressed to $c_{1}$ and $d_{2}$ is processed by delta encoding with $d_{1}$ as the reference, leading to the delta $\Delta_{1,2}$. Let $s_{\mathcal{u}}$ denote the free space in the physical page. Upon another overwrite with the content $d_{3}$, we calculate two deltas: $\Delta_{1,3}$ with $d_{1}$ as the reference, and $\Delta_{2,3}$ with $d_{2}$ as the reference. We also compress the block content $d_{3}$ to $c_{3}$. Dependent upon the size of $\Delta_{1,3}, \Delta_{2,3}$, and $c_{3}$ and $s_{u}$, we have

- If $\left|\Delta_{1,3}\right|$ is the smallest, then we discard $\Delta_{1,2}$ and append $\Delta_{1,3}$ after $c_{1}$, as shown in Fig. 7(b).

- If $\left|\Delta_{2,3}\right|$ is the smallest, then we append $\Delta_{2,3}$ after $\Delta_{1,2}$, as shown in Fig. 7(c).

- If either $\left|c_{3}\right|$ or $\left|s_{u}\right|$ is the smallest, we discard $d_{1}$ and $\Delta_{1,2}$ altogether, and write $c_{3}$ into the physical page, as shown in Fig. 7(d).

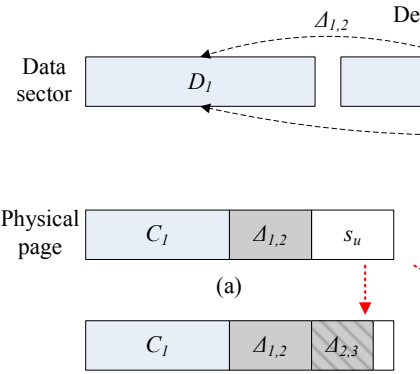

(c)
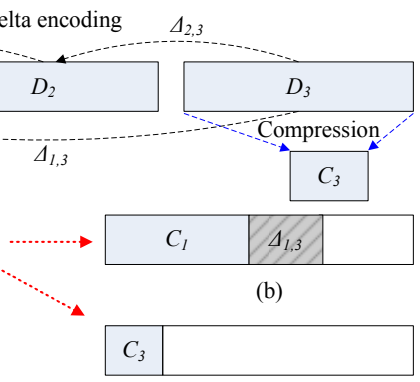

(d)

Figure 7: An example to illustrate intra-page delta encoding.

When being written into physical pages, each delta also attaches a small header that contains all the necessary metadata (e.g., pointer to its reference, and the length of the delta).

\subsection{Memory Usage}

This subsection discusses the DRAM usage inside block devices in support of built-in compression. Block devices keep an LBA-PBA mapping table in their DRAM. The number of table entries equals to the number of LBAs being exposed by block devices. As discussed above, each compressed block is stored together with its header within one physical page. After reading one physical page, we can use the headers to identify and locate different compressed blocks within the physical page. Therefore, each mapping table entry only holds the corresponding PBA and does not store the intra-page location information. The number of bits representing each PBA depends on the physical storage capacity, e.g., with $4 \mathrm{kB}$ block size, 4 bytes per PBA is enough for block devices with no more than 16TB physical storage capacity.

Block devices use a physical page property table to record the runtime usage information of each physical page. Each table entry uses 3 bytes and consists of (1) a 1-bit hotness flag to indicate whether the physical page contains any hot LBA, (2) a 1-bit hole flag to indicate whether the physical page contains a hole left by a stale compressed block, (3) a 10-bit field to record the size of the unused space at the tail of the physical page, (4) a 6-bit field to record the total number of blocks in the physical page, and (5) a 6-bit field to record the number of stale blocks in the physical page. If the last two 6-bit fields are equal, then the physical page does not contain any valid data and can be directly reused.

The content of LBA-PBA mapping table and physical page property table is periodically check-pointed to NVM. Since the header of each block incorporates all the necessary metadata, we can carry out a full-scan to reconstruct these two tables (except the hotness flag in the physical page property table) in the case of complete table corruption. Let $C_{P}$ denote the number of physical pages inside the block device, and $C_{L}$ denote the number of LBAs exposed by the block device. We can estimate the size of the two tables as $C_{L} \cdot 4 \mathrm{~B}$ and $C_{P} \cdot 3 \mathrm{~B}$, respectively. For example, if the block device contains $256 \mathrm{~GB}$ of physical space (i.e., $C_{P}$ is $64 \mathrm{M}$ ) and exposes $1 \mathrm{~TB}$ of storage space (i.e., $C_{L}$ is $256 \mathrm{M}$ ), the LBA-PBA mapping table and physical page property table occupy $1 \mathrm{~GB}$ and $192 \mathrm{MB}$ memory, respectively.

\section{EVALUATION}

\subsection{Experiment Setup}

To facilitate the evaluation, we implemented an NVM-based block device simulator based upon the Microsoft Research SSD model [1] for the Disksim 4.0 simulation environment [3]. We modified the SSD model to support byte-addressability (both write and read) of NVM, and incorporated a $16 \mathrm{MB}$ write buffer module. It stripes each $4 \mathrm{kB}$ data block across two channels to further reduce the read latency. This work uses an 8-channel architecture and sets the NVM sensing latency of each $4 \mathrm{kB}$ block as only $2 \mu$ s. Therefore, the simulated block device can achieve up to $8 \mathrm{~GB} / \mathrm{s}$ internal NVM read throughput, which can saturate the PCIe Gen $3 \times 8$ interface. We consider three per- $4 \mathrm{kB}$ NVM write latency: $4 \mu \mathrm{s}, 10 \mu \mathrm{s}$ and $20 \mu \mathrm{s}$, which correspond to NVM write vs. read latency ratio of 2:1, 5:1 and 10:1, respectively.

To evaluate the block device speed performance, we use both synthetic workloads and real-world I/O traces. The synthetic workloads consist of random $4 \mathrm{kB}$ access under different queue depth (hence different IOPS). We consider both read-only workload and mixed read/write workloads with $70 \%: 30 \%$ of read vs. write ratio. For trace-based evaluation, we used six popular traces collected 


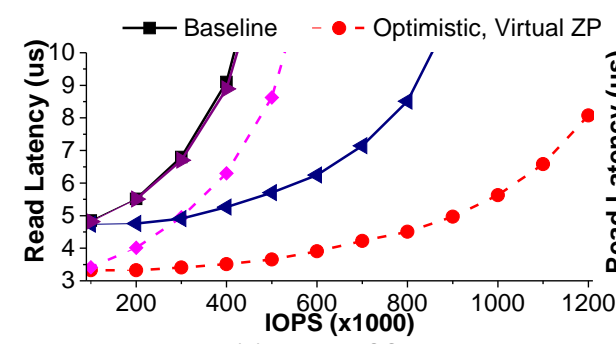

(a) $R_{c}=0.2, S S F=3$

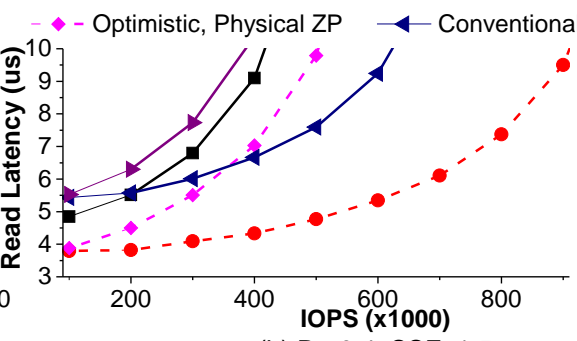

(b) $R_{c}=0.4, S S F=1.5$

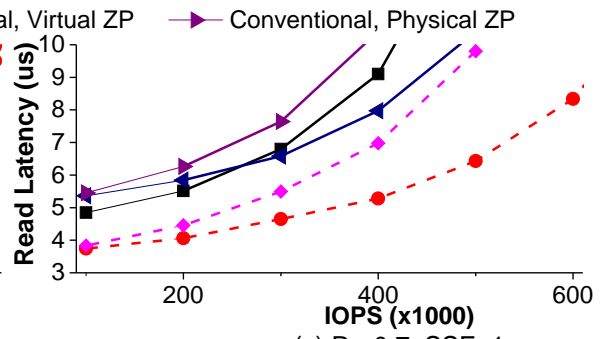

(c) $R_{c}=0.7, S S F=1$

Figure 8: Read latency vs. IOPS of different combinations of decompression and zero-padding options under random $4 \mathrm{kB}$ $70 \%$-read workloads. The NVM write vs. read latency ratio is 5:1 (4kB NVM sensing latency is $2 \mu$ s).

from FIU [15] and MSR Cambridge enterprise servers [23]: web-vm, homes, rsrch_2, proj_3, stg_1, src2_1. The read request percentages of the chosen traces are $26.7 \%, 27.4 \%, 64.3 \%, 87.4 \%, 93 \%$ and $99.5 \%$.

We assume the compression ratio follows a Gaussian distribution $\mathcal{N}\left(\mu, \sigma^{2}\right)$ where the variance is $10 \%$ of the mean (i.e., $\sigma^{2}=$ $0.1 \cdot \mu)$ [37]. Let $R_{c}$ denote the mean of the compression ratio, we consider three different values of $R_{c}$ (i.e., $0.2,0.4$ and 0.7 ). Regarding the intra-page delta-encoding, we also assume the delta-encoding compression ratio follows Gaussian distribution where the variance is $10 \%$ of the mean [37]. Let $R_{\text {delta }}$ denote the mean of deltaencoding ratio, we consider three different values of $R_{\text {delt a }}$ (i.e., $0.01,0.1$ and 0.3 ).

Since the read latency of each physical page depends on how heavily compressed blocks are packed into this physical page, there is a speed performance vs. storage saving trade-off design space. To reveal such trade-off, we define the storage saving factor (SSF) as the ratio between the LBA space and PBA space of block devices. As we increase the SSF, we may improve the bit cost reduction at the penalty of longer read latency. In our experiments, we consider SSF of $1,2,3$, and 4 when the mean of compression ratio $R_{c}$ is 0.2 , SSF of $1,1.5,2$ when $R_{c}$ is 0.4 , and SSF of 1 when $R_{c}$ is 0.7 .

\subsection{Effectiveness of Optimistic Data Decompression and Zero-padding}

We carried out simulations to cohesively evaluate the effectiveness of the proposed optimistic decompression and zero-padding with punctured ECC. As discussed in Section 2.3, given the per-4kB NVM sensing latency of $2 \mu \mathrm{s}$, the $4 \mathrm{kB} \mathrm{BCH}$ decoding contributes another $2 \mu$ s to the total read latency. If we do not use the proposed optimistic data decompression, data decompression will contribute additional $\lambda \cdot 2 \mu$ s latency (where $\lambda<1$ denotes the compression ratio). Once we use the proposed optimistic data decompression, data decompression no longer contributes to the total read latency. Regarding zero-padding punctured ECC, we consider both the physical zeropadding and the virtual zero-padding, as discussed in Section 3.2. Let $l_{u}$ denote the length of the unused space in one physical page. When using zero-padding punctured ECC, the latency of NVM sensing and ECC decoding will both reduce to $\frac{4 \mathrm{kB}-l_{u}}{4 \mathrm{kB}} \cdot 2 \mu \mathrm{s}$.

For the purpose of comparison, we carried out experiments under five different scenarios, including the baseline that does not have built-in compression, and four different combinations of decompression (conventional vs. optimistic decompression) and zero-padding
(ZP) (physical vs. virtual ZP). Fig. 8 shows the read latency vs. IOPS of different scenarios under three different combinations of $R_{c}$ (i.e., mean of the compression ratio) and SSF (i.e., the storage saving factor), where the workload is mixed read/write with $70 \%$ read. The results show that the proposed optimistic data decompression significantly outperforms the conventional decompression, because decompression latency starts to play an important role in the presence of very short NVM sensing latency. Meanwhile, the virtual ZP significantly outperforms physical ZP, because virtual ZP can reduce the write traffic and hence reduce the write-to-read interference. Moreover, the results show that the read latency difference becomes larger as the IOPS increases due to the more significant queuing effect. For example, under $R_{c}=0.2$ and $\mathrm{SSF}=3$, the combination of optimistic decompression and virtual $\mathrm{ZP}$ can reduce the latency by over $95 \%$ at $600 \mathrm{~K}$ IOPS, compared with the baseline.

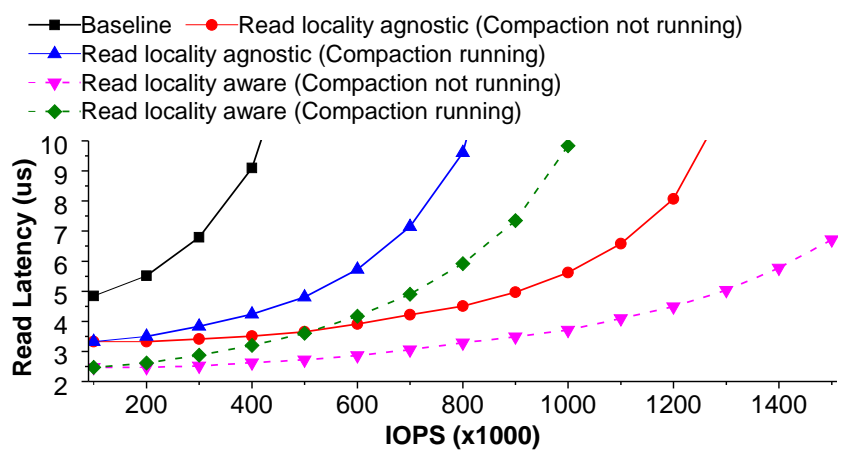

Figure 9: Read latency vs. IOPS when using different compaction approaches under random $4 \mathrm{kB} 70 \%$-read workloads. $R_{c}$ is 0.2 and SSF is 3 . The NVM write vs. read latency ratio is $5: 1$ (4kB NVM sensing latency is $2 \mu \mathrm{s}$ ).

\subsection{Impact of Compaction}

We further studied the impact of compaction and effectiveness of the proposed read locality aware compaction. Fig. 9 compares the read latency vs. IOPS with and without using the read locality aware compaction, where we set $R_{c}$ as 0.2 and SSF as 3 . Since the read latency could noticeably degrade when the background compaction is running, Fig. 9 shows the results for both cases when the compaction is or is not running. When compaction is running, we set 


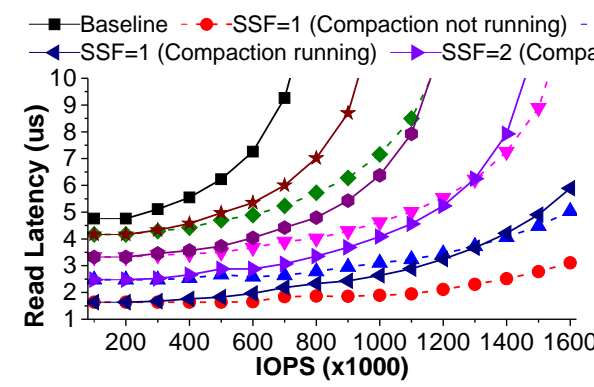

(a) NVM write/read latency ratio 2:1

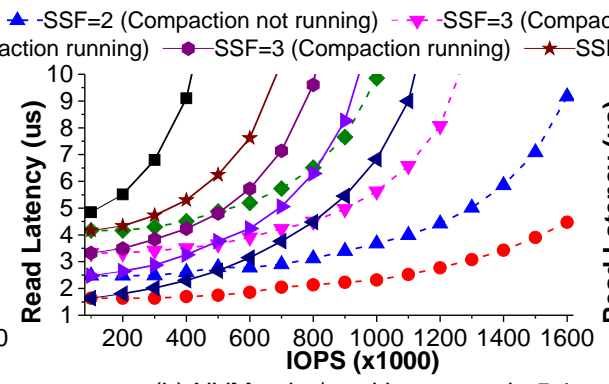

(b) NVM write/read latency ratio 5:1

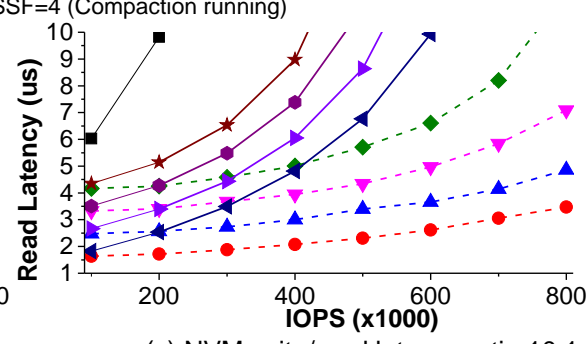

(c) NVM write/read latency ratio 10:1

Figure 10: Read latency vs. IOPS under random $4 \mathrm{kB} 70 \%$-read workload with $R_{c}=0.2(4 \mathrm{kB} \mathrm{NVM}$ sensing latency is $2 \mu \mathrm{s})$.

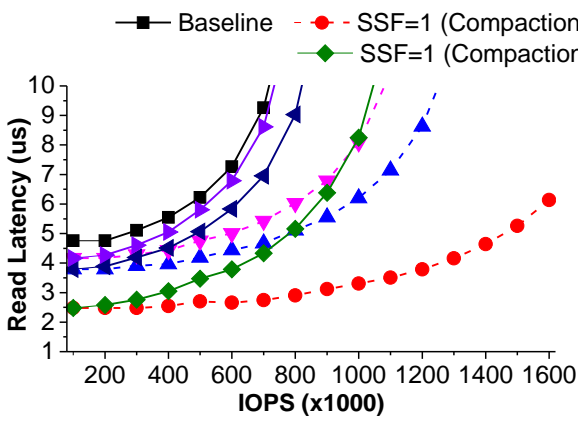

(a) NVM write/read latency ratio 2:1

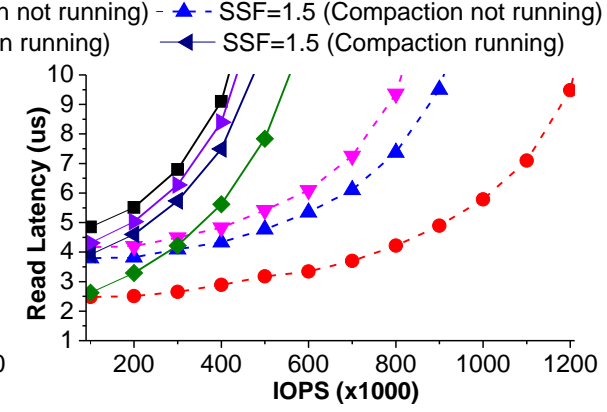

(b) NVM write/read latency ratio 5:1

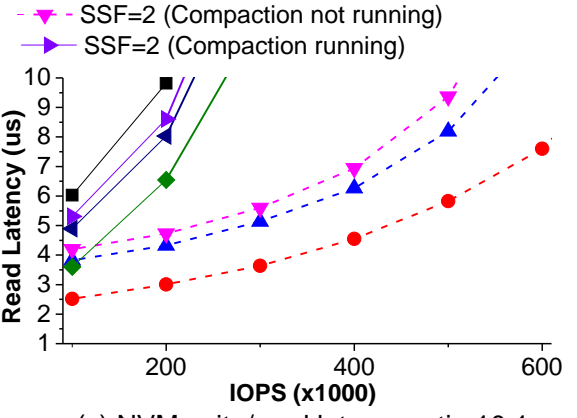

(c) NVM write/read latency ratio 10:1

Figure 11: Read latency vs. IOPS under random $4 \mathrm{kB} 70 \%$-read workload with $R_{c}=0.4$ (4kB NVM sensing latency is $\left.2 \mu \mathrm{s}\right)$.

that the compaction carries out one selective data merging operation every two host-issued write requests. We use Rosenblum's hot/cold data model $[9,29]$ to emulate the read locality. We set that $10 \%$ of PBAs are access-hot and serve $80 \%$ of all the read requests. The baseline does not have built-in compression. All the cases (except baseline) use optimistic data decompression and virtual zero-padding. The results show that the proposed read locality aware compaction noticeably outperforms the locality agnostic compaction. Under $800 \mathrm{~K}$ IOPS, compared with locality agnostic compaction, it reduces the read latency by $26.9 \%$ when compaction is not running, and $38.4 \%$ when compaction is running.

\subsection{Cost vs. Performance Trade-off}

The proposed design framework essentially enables a cost vs. latency performance trade-off space. As we pack more compressed blocks in each physical page, we can further reduce the effective bit cost, but the read latency will degrade. To demonstrate such a trade-off space, we further carried out experiments under many different configurations.

1) Synthetic Workload Results: Fig. 10 and Fig. 11 show the tradeoffs with different NVM write vs. read latency ratios and SSF when the mean of data compression ratio $R_{c}$ is 0.2 and 0.4 , respectively. Mixed random $4 \mathrm{kB}$ workloads with $70 \%$ read are used. The results clearly show that, as we reduce the bit cost by increasing the SSF, the read latency increases. In spite of the large trade-off space, all the configurations can achieve (much) shorter read latency than the baseline. Moreover, the read latency gain over the baseline becomes larger as the NVM write vs. read latency ratio increases.
Fig. 12 shows the read latency reduction compared with the baseline under random $4 \mathrm{kB} 100 \%$-read workloads, which clearly reveals the storage saving vs. read latency trade-off and benefits of the proposed solutions as well. As shown in Fig. 12(a), with $R_{c}$ of 0.2 , the read latency reduces by $86.6 \%, 79.6 \%, 68.6 \%$, and $51.2 \%$ when the SSF is $1,2,3$, and 4 at $800 \mathrm{~K}$ IOPS, compared with the baseline. We also carried out evaluations on write latency. Due to the use of internal SRAM/DRAM write buffer with power loss protection, write latency will remain constant unless the write buffer becomes full due to high IOPS and compaction-induced overhead. As pointed out above, we assume $16 \mathrm{MB}$ write buffer. Fig. 13 shows the write latency when the mean of data compression ratio $R_{c}$ is 0.2 and 0.4 , respectively. The write latency is relatively stable $(\sim 4.5 \mu \mathrm{s})$ before reaching the peak IOPS. After reaching the peak IOPS, the write latency increases exponentially due to large queuing delay.

2) Real-world I/O Trace Results: Fig. 14 and Fig. 15 show results with different SSF under different traces when $R_{c}$ is 0.2 and 0.4 . Fig. 14(a) and Fig. 15(a) show the results when we simply replayed the original traces (at $1 \times$ intensity). Fig. 14(b) and Fig. 15(b) show the results under much higher stress by shrinking the request dispatch time in each trace by $50 \times$. The results clearly show the trade-offs and the benefits of proposed solutions. Moreover, read latency reduction over the baseline improves as the request intensity increases. For example, under $R_{c}=0.2$ and $\mathrm{SSF}=3$, the read latency reduction ranges from $26.2 \%$ to $41.5 \%$ at $1 \times$ intensity, and from $36.8 \%$ to $65.1 \%$ at $50 \times$ intensity for all the traces. Results show that write latency remains almost the same as the baseline, even for the write-domiant traces (i.e., web-vm and homes) under $50 \times$ intensity. 


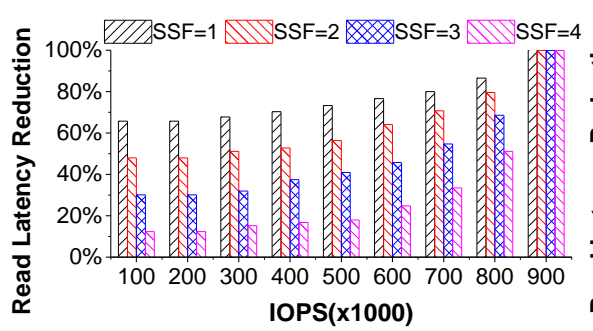

(a) $R_{c}=0.2$

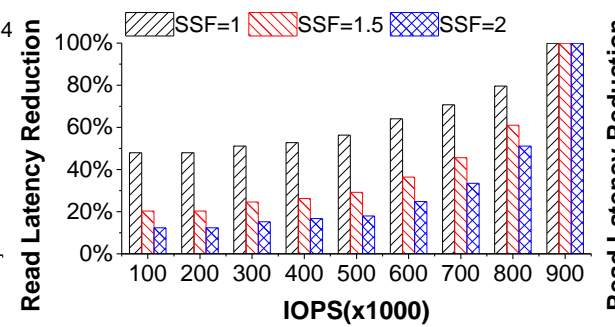

(b) $R_{c}=0.4$

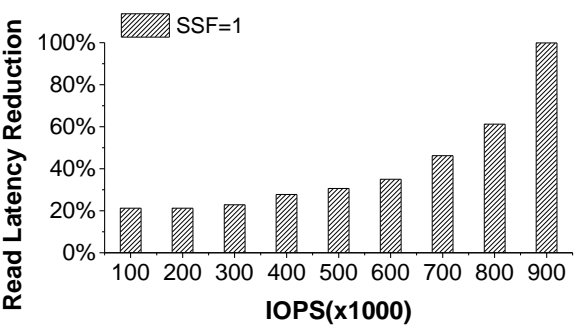

(c) $R_{c}=0.7$

Figure 12: Read latency reduction (higher is better) over baseline vs. IOPS under random 4kB $100 \%$-read workloads (4kB NVM sensing latency is $2 \mu \mathrm{s}$ ).

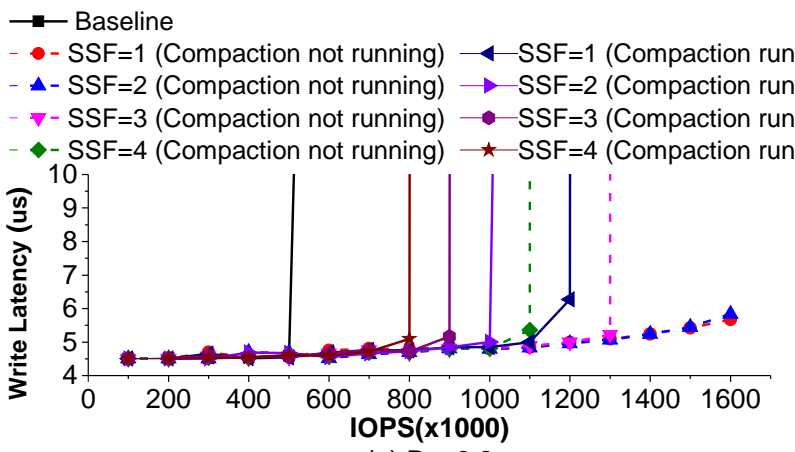

(a) $\mathrm{R}_{\mathrm{C}}=0.2$

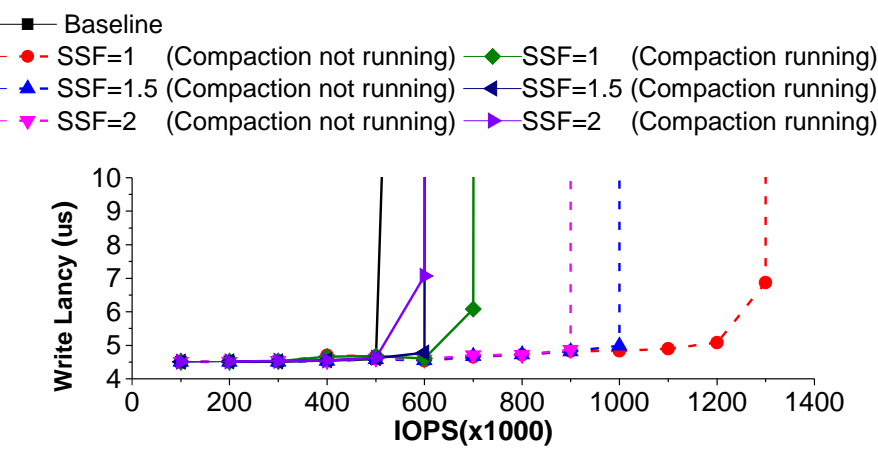

(b) $R_{C}=0.4$

Figure 13: Write Latency under random $4 \mathrm{kB} 70 \%$-read workload. The NVM write vs. read latency ratio is $5: 1$ (4kB NVM sensing latency is $2 \mu \mathrm{s})$.

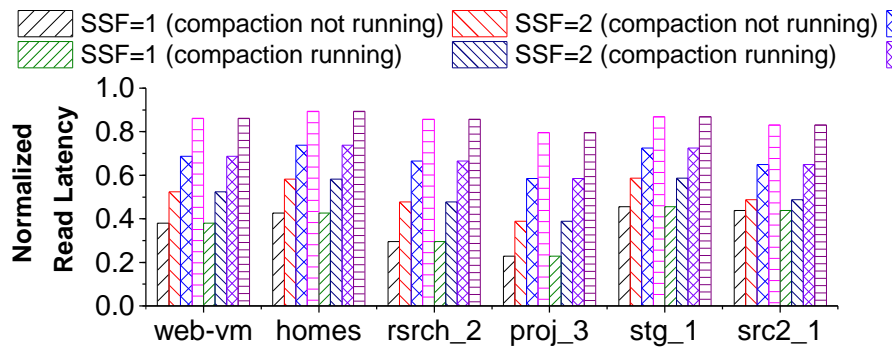

(a) $1 \mathrm{x}$ intensity

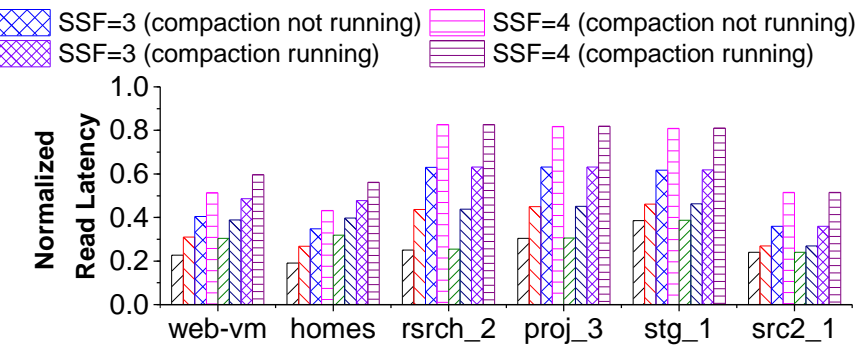

(b) 50x intensity

Figure 14: Normalized Read Latency (lower is better) over baseline under different real-world $\mathrm{I} / \mathrm{O}$ traces with $R_{c}=0.2$. The NVM write vs. read latency ratio is $5: 1$ (4kB NVM sensing latency is $2 \mu \mathrm{s})$.

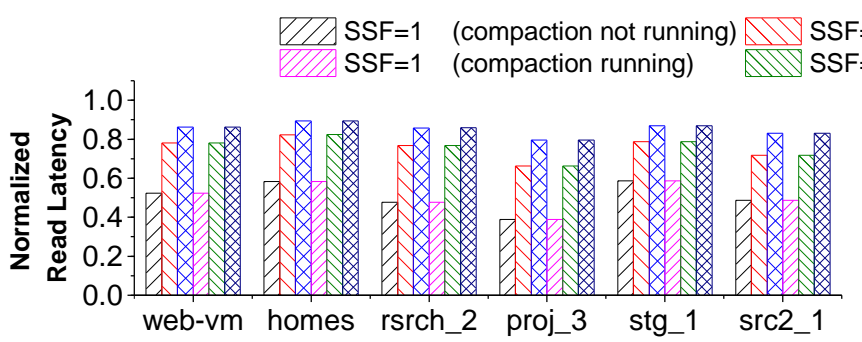

(a) $1 \mathrm{x}$ intensity
$\mathrm{SSF}=1.5$ (compaction not running) SSF=2 (compaction not running) $\mathrm{SSF}=1.5$ (compaction running) $\mathrm{SSF}=2$ (compaction running)

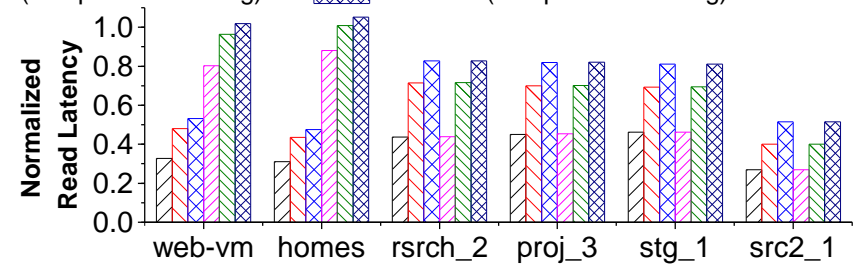

(b) 50x intensity

Figure 15: Normalized Read Latency (lower is better) over baseline under different real-world I/O traces with $R_{c}=0.4$. The NVM write vs. read latency ratio is $5: 1$ (4kB NVM sensing latency is $2 \mu$ s). 


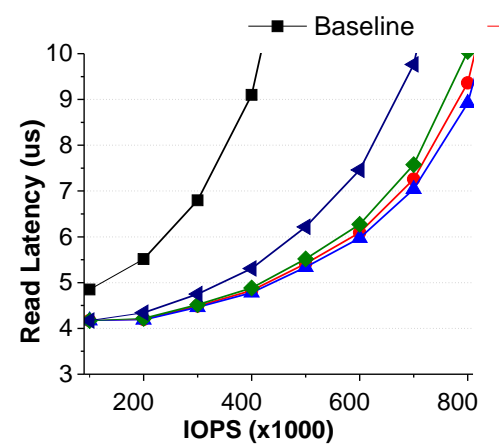

(a) $R_{c}=0.4, \mathrm{SSF}=2$.

NVM write/read latency ratio is $5: 1$

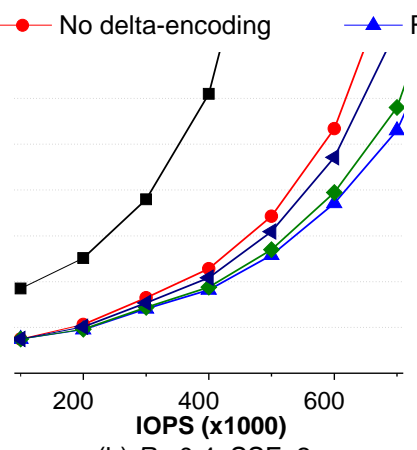

(b) $R_{c}=0.4, \mathrm{SSF}=2$

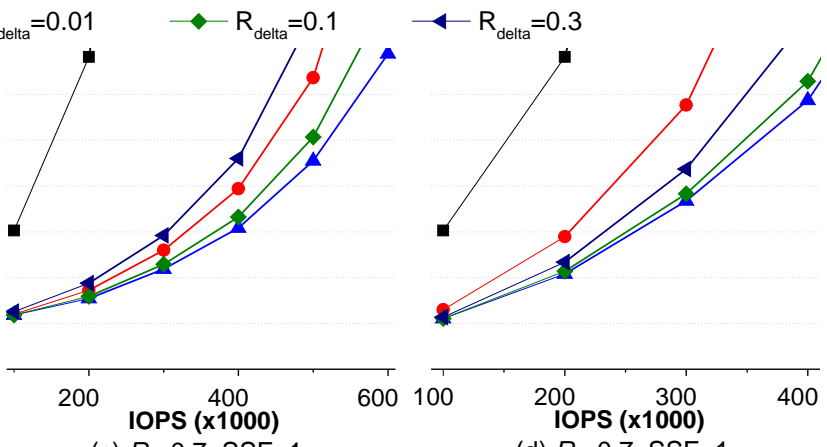

(c) $R_{c}=0.7, \mathrm{SSF}=1$ (d) $R_{c}=0.7, \mathrm{SSF}=1$.

NVM write/read latency ratio is 10:1 NVM write/read latency ratio is 5:1 NVM write/read latency ratio is $10: 1$

\subsection{Effectiveness of Delta Encoding}

We finally evaluated the effectiveness of the proposed intra-page delta encoding method. Although this method can reduce the NVM write traffic, it has to first fetch the old content from NVM, leading to additional read traffic. The net effect on the overall block device read latency depends on the delta-encoding compression ratio, workload characteristics, and NVM write vs. read latency ratio. Fig. 16 shows the impact of delta-encoding under different cases. The baseline case does not have built-in compression, and the no delta-encoding case only uses optimistic data decompression and virtual $\mathrm{ZP}$. The other cases use delta-encoding in addition to optimistic data decompression and virtual ZP. Compaction is not running in all the cases. We assume that $50 \%$ of write can be handled by delta-encoding with the delta-encoding compression ratio of $0.01,0.1$, and 0.3 , respectively. The results show that the benefit of delta-encoding noticeably increases when the NVM write latency becomes relatively longer. For example, with NVM write vs. read latency ratio of 10:1, the cases with delta-encoding consistently outperforms the case without delta-encoding. Meanwhile, the results show that the use of delta-encoding could degrade the block device read latency performance under relatively small NVM write vs. read latency ratio (e.g., 5:1).

\section{RELATED WORK}

Prior work has well studied the application of data reduction schemes (including compression, deduplication, and delta encoding) to flashbased block devices. In [19, 27, 36], several techniques were developed to design and implement flash translation layer (FTL) that can support transparent compression. The authors of [21] studied the use of compression to solely enhance the flash memory cycling endurance. The authors of [39] compared the implementation of compression at the application, file system and FTL levels. Design techniques presented in [8] enable host-side database compression by leveraging new FTL primitives. The authors of $[5,11,14]$ developed a variety of design techniques that reduce the cost of implementing transparent data deduplication inside flash-based block devices. In the context of applying delta encoding to flash-based block devices, the authors of [31,37] solely focused on exploiting the content similarity of data overwrite, while the authors of [26] extended to searching for similarity among data blocks at different LBAs. Prior work [18, 20, 22] also studied the combination of different data reduction schemes for flash-based block devices. Nevertheless, the inherent drawbacks of NAND flash memory (in particular the lack of in-place update and fine-grained data accessibility) make it fundamentally difficult to fully exploit the potential of data reduction schemes. Due to the characteristics of flash memory, the compression can only reduce the bit cost, and their corresponding read latency can only be worse than the case of without using compression. To the best of our knowledge, there is no prior work that studied NVM-based block device with built-in compression. This work develops specific techniques that cohesively exploit NVM byte accessibility, data compressibility, and ECC coding theory to simultaneously reduce bit cost and read latency of NVM-based block devices without compromising storage reliability.

The authors of $[10,13,30,33]$ investigated the use of compression to reduce write traffic and extend the lifetime of NVM-based main memory. The authors of [24] integrated compression with expansion coding to improve write latency, energy and lifetime of MLC/TLC NVM-based main memory. The techniques presented in [13] can reduce both the write latency and read latency by storing highly compressible data in SLC form for MLC PCM based memory system. These prior work foucs on NVM-based main memory and does not address the combination of compression and long ECC codeword in block devices. In addition, they can not simultaneously reduce cost and improve performance.

\section{CONCLUSION}

This paper presented a set of design techniques that can minimize the read latency of NVM-based block devices with built-in transparent data compression. Leveraging the operational characteristics of ECC and data decompression, we first developed a technique that can eliminate decompression-induced read latency penalty on the read data path. We further developed a technique that can reduce the NVM physical page read latency by exploiting the fine-grained NVM accessibility and ECC coding theory. A read locality aware compaction strategy is further developed to reduce block device read latency by taking advantage of the abundant read locality in real-world applications. Finally, we developed an intra-page delta 
encoding scheme to exploit data overwrite content similarity in block devices with built-in data compression. To quantitatively evaluate the developed design techniques, we implemented an NVMbased block device simulator and accordingly carried out extensive simulations. Results show that, by deploying the developed design techniques, we not only can reduce bit cost but also can achieve (much) lower read latency, compared with block devices without built-in data compression.

\section{ACKNOWLEDGMENTS}

The authors would like to thank the anonymous reviewers for their constructive comments. This work was supported in part by the U.S. National Science Foundation under Grants CCF-1629218 and CCF-1629201.

\section{REFERENCES}

[1] Agrawal, N., Prabhakaran, V., Wobber, T., Davis, J. D., Manasse, M., and PANIGRAhy, R. Design tradeoffs for SSD performance. In Proceedings of USENIX Annual Technical Conference (ATC) (2008), pp. 57-70.

[2] Blahut, R. E. Theory and Practice of Error Control Codes. Addison Wesley, 1984

[3] Bucy, J. S., Schindler, J., Schlosser, S. W., And Ganger, G. R. The DiskSim Simulation Environment Version 4.0 Reference Manual . CMU Parallel Data Lab Technical Report CMU-PDL-08-101, 2008.

[4] Campello, D., Lopez, H., Koller, R., Rangaswami, R., and Useche, L. Nonblocking writes to files. In Proceedings of USENIX Conference on File and Storage Technologies (FAST) (2015), pp. 151-165.

[5] Chen, F., Luo, T., AND ZhANG, X. CAFTL: A content-aware flash translation layer enhancing the lifespan of flash memory based solid state drives. In Proceedings of USENIX Conference on File and Storage Technologies (FAST) (2011), pp. 77-90.

[6] Chiang, M., Lee, P. C. H., And Chang, R. Using data clustering to improve cleaning performance for flash memory. Software: Practice and Experience 29, 3 (March 1999).

[7] CHo, S., AND LEe, H. Flip-N-Write: A simple deterministic technique to improve PRAM write performance, energy and endurance. In Proceedings of IEEE/ACM International Symposium on Microarchitecture (MICRO) (2009), pp. 347-357.

[8] Das, D., Arteaga, D., Talagala, N., Mathiasen, T., And Lindström, J. NVM compression-hybrid flash-aware application level compression. In Workshop on Interactions of NVM/Flash with Operating Systems and Workloads (INFLOW) (2014).

[9] Desnoyers, P. Analytic models of ssd write performance. ACM Transactions on Storage (TOS) vol. 10, no. 2 (2014), p. 25.

[10] Guo, Y., HuA, Y., AND Zuo, P. DFPC: A dynamic frequent pattern compression scheme in NVM-based main memory. In Proceedings of the Conference on Design, Automation \& Test in Europe (2018), pp. 1622-1627.

[11] Gupta, A., Pisolkar, R., Urgaonkar, B., AND Sivasubramaniam, A. Leveraging value locality in optimizing NAND flash-based SSDs. In Proceedings of USENIX Conference on File and Storage Technologies (FAST) (2011), pp. 91-103.

[12] Hsien, J.-W., Kuo, T.-W., AND Chang, L.-P. Efficient identification of hot data for flash memory storage systems. ACM Transactions on Storage (TOS) vol. 2, no. 1 (2006), pp. 22-40.

[13] JiAng, L., Zhao, B., Zhang, Y., YANG, J., AND Childers, B. R. Improving write operations in MLC phase change memory. In Proceedings of IEEE International Symposium on High Performance Computer Architecture (HPCA) (2012), pp. 1-10.

[14] Kim, J., Lee, C., Lee, S., Son, I., ChoI, J., Yoon, S., LeE, H.-U., Kang, S., Won Y., AND CHA, J. Deduplication in SSDs: Model and quantitative analysis. In Proceedings of the IEEE Symposium on Mass Storage Systems and Technologies (MSST) (2012), pp. 1-12.

[15] Koller, R., AND RANGASWAmi, R. I/O deduplication: Utilizing content similarity to improve I/O performance. ACM Transactions on Storage (TOS) vol. 6, no. 3 (2010), p. 13.

[16] Lee, B. C., Ipek, E., Mutuu, O., And Burger, D. Architecting phase change memory as a scalable DRAM alternative. In Proceedings of the 36th Annual International Symposium on Computer Architecture (ISCA) (2009), pp. 2-13.

[17] LEE, J., AND KIM, J.-S. An empirical study of hot/cold data separation policies in solid state drives (SSDs). In Proceedings of the International Systems and Storage Conference (SYSTOR) (2013), pp. 12:1-12:6.

[18] LeE, S., Kim, T., PARK, J.-S., AND KIM, J. An integrated approach for managing the lifetime of flash-based SSDs. In Proceedings of the Conference on Design, Automation and Test in Europe (2013), pp. 1522-1525.

[19] Lee, S., Park, J., Fleming, K., Kim, J., ET Al. Improving performance and lifetime of solid-state drives using hardware-accelerated compression. IEEE Transactions on Consumer Electronics vol. 57, no. 4 (2011).

[20] Li, C., Shilane, P., Douglis, F., Shim, H., Smaldone, S., and Wallace, G. Nitro: A capacity-optimized SSD cache for primary storage. In Proceedings of USENIX Annual Technical Conference (ATC) (2014), pp. 501-512.

[21] Li, J., Zhao, K., Zhang, X., Ma, J., Zhao, M., AND Zhang, T. How much can data compressibility help to improve NAND flash memory lifetime? In Proceedings of USENIX Conference on File and Storage Technologies (FAST) (2015), pp. 227-240.

[22] Li, W., Jean-Baptise, G., Riveros, J., Narasimhan, G., Zhang, T., and Zhao, M. Cachededup: In-line deduplication for flash caching. In Proceedings of USENIX Conference on File and Storage Technologies (FAST) (2016), pp. 301-314.

[23] Narayanan, D., Donnelly, A., And Rowstron, A. Write off-loading: Practical power management for enterprise storage. ACM Transactions on Storage (TOS) vol. 4, no. 3 (2008), p. 10.

[24] Palangappa, P. M., And Mohanram, K. CompEx: Compression-expansion coding for energy, latency, and lifetime improvements in MLC/TLC NVM. In Proceedings of IEEE International Symposium on High Performance Computer Architecture (HPCA) (2016), pp. 90-101.

[25] Park, D., AND Du, D. H. C. Hot data identification for flash-based storage systems using multiple bloom filters. In Proceedings of the IEEE Symposium on Mass Storage Systems and Technologies (MSST) (2011), pp. 1-11.

[26] PARK, J., LEE, S., AND KIM, J. DAC: dedup-assisted compression scheme for improving lifetime of NAND storage systems. In Proceedings of the Conference on Design, Automation \& Test in Europe (2017), pp. 1249-1252

[27] PARK, T., AND Kim, J.-S. Compression support for flash translation layer. In Proceedings of the International Workshop on Software Support for Portable Storage (2010), pp. 19-24.

[28] Qureshi, M. K., Srinivasan, V., And Rivers, J. A. Scalable high performance main memory system using phase-change memory technology. In Proceedings of the 36th Annual International Symposium on Computer Architecture (ISCA) (2009), pp. 24-33.

[29] Rosenblum, M., and Ousterhout, J. K. The design and implementation of a $\log$-structured file system. ACM Transactions on Computer Systems (TOCS) vol. 10, no. 1 (1992), 26-52.

[30] Sun, G., Niu, D., OuYAng, J., AND Xie, Y. A frequent-value based PRAM memory architecture. In Proceedings of the 16th Asia and South Pacific Design Automation Conference (ASP-DAC) (2011), pp. 211-216.

[31] WU, G., AND HE, X. Delta-FTL: improving SSD lifetime via exploiting content locality. In Proceedings of the 7th ACM European Conference on Computer Systems (EuroSys) (2012), pp. 253-266.

[32] Xu, C., Niu, D., Muralimanohar, N., Balasubramonian, R., Zhang, T., Yu, S., AND XIE, Y. Overcoming the challenges of crossbar resistive memory architectures. In Proceedings of the IEEE 21st International Symposium on High Performance Computer Architecture (HPCA) (2015), pp. 476-488

[33] Xu, J., Feng, D., Hua, Y., Tong, W., Liu, J., And Li, C. Extending the lifetime of NVMs with compression. In Proceedings of the Conference on Design, Automation \& Test in Europe (2018), pp. 1604-1609.

[34] Xu, J., AND Swanson, S. NOVA: A log-structured file system for hybrid volatile/non-volatile main memories. In Proceedings of USENIX Conference on File and Storage Technologies (FAST) (2016), pp. 323-338.

[35] Xu, W., Liu, J., AND Zhang, T. Data manipulation techniques to reduce phase change memory write energy. In Proceedings of the ACM/IEEE International Symposium on Low Power Electronics and Design (ISLPED) (2009), pp. 237-242.

[36] Y.m, K. S., BAHN, H., AND КоH, K. A flash compression layer for smartmedia card systems. IEEE Transactions on Consumer Electronics vol. 50, no. 1 (2004), 192-197.

[37] Zhang, X., Li, J., Wang, H., Zhao, K., And Zhang, T. Reducing solid-state storage device write stress through opportunistic in-place delta compression. In Proceedings of USENIX Conference on File and Storage Technologies (FAST) (2016), pp. 111-124.

[38] Zhou, P., Zhao, B., Yang, J., And Zhang, Y. A durable and energy efficient main memory using phase change memory technology. In Proceedings of the 36th Annual International Symposium on Computer Architecture (ISCA) (2009), pp. 14-23.

[39] Zuck, A., Toledo, S., Sotnikov, D., And Harnik, D. Compression and SSDs: Where and how? In Workshop on Interactions of NVM/Flash with Operating Systems and Workloads (INFLOW) (2014). 